\title{
The advantage of MRI in detection of Coincidental adenocarcinoma of palate and pleomorphic adenoma of parotid - a case report
}

\author{
Sleem $\mathrm{H}^{\mathrm{a},{ }^{*}}$, Sharabasy $\mathrm{I}^{\mathrm{b}}$ and Foad $\mathrm{R}^{\mathrm{c}}$ \\ ${ }^{a} B D S$, Ms, Phd, MOS, MOMS. Associate professor of oral and maxillofacial surgery, faculty of dentistry Ain shams university. \\ ${ }^{b} B D S, M s, M O M S, A B O M S$. Researcher oral and maxillofacial surgery, faculty of dentistry Ain shams university. \\ ${ }^{c} B D S, M s$, Phd. Lecturer of oral pathology faculty of dentistry Ain shams university.
}

\begin{abstract}
Salivary gland tumors account for fewer than 3\% of the head and neck tumors. Typically salivary gland neoplasm presented as a single swelling localized to one salivary gland. A more unusual event is the development of multiple neoplasms located either in different glands or in one gland. This manuscript present a case of palatal swelling diagnosed by incisional biopsy as polymorphous adenocarcinoma (PAC). Magnetic resonance imaging (MRI) examination revealed another parotid lesion which was diagnosed as pleomorphic adenoma (PA). Palatal mass was resected with safety margin; superficial parotidectomy was also done for parotid lesion sparing facial nerve. Post-operative radiotherapy was recommended by pathologist due to evident perineural invasion of PAC. The patient is tumor free after one year follow-up. The present case has more than one risk factor which favors expectation of multiple tumors and mandate examination of all salivary tissues. In case of multiple salivary tumors each tumor should be diagnosed and treated separately according to its nature and extension.
\end{abstract}

Keywords: Salivary gland; tumors; parotid; palate; MRI

\section{INTRODUCTION}

Salivary gland tumors account for less than $3 \%$ of head and neck tumors ${ }^{[1]}$. Many case reports describes presence of more than one salivary gland tumor either synchronously or metachronusly ${ }^{[2,3]}$. Several taxonomies are used to describe synchronous/metachronous occurrence of salivary gland neoplasms. Multiple salivary tumors may be considered from three standpoints: according to incidence time (synchronous or metachronous ), anatomical aspect (unilateral or bilateral), and histological aspect (identical or different pathology). Multiple malignant salivary tumors of identical pathology are much more common, which proposes the possibility of genetic risk factor, rather than only accidental event ${ }^{[4]}$. Polymorphous adenocarcinoma (PAC) was first described in 1984, diagnoses is usually made based on the hematoxylin and eosin morphology. Solid nests of monotonous epithelial cells arranged in cribriform and trabecular masses. Focal papillary cystic areas can typically be present within the same lesion. The epithelial

*Corresponding author: Sleem, Heba Abdulwahed, Department of Oral and Maxillofacial Surgery Ain Shams University, Cairo, Egypt.

E-mail: drsleemh@gmail.com

Received: 15 August 2019 Accepted: 20 September 2019 cells are usually cuboidal, columnar or spindle with eosinophilic cytoplasm. The nuclei are uniform, round or ovoid, with a characteristic "washed out" appearance. Mucous cells, clear cells or oncocytic cells may also be seen. The concentric whorling is noticed around regional neurovascular bundles "a targetoid appearance", this perineural invasion (PNI) or neurotropism is a distinguishing feature of PAC and can be recognized in 30\% of cases. While perineural involvement is common, mitotic figures are uncommon and necrosis is seen in high-grade transformation ${ }^{[5]}$.

PAC has a strong predilection to minor salivary glands of the palate. It tends to have lower local recurrence rates and lower metastatic potential however, PAC is invasive and locally destructive. Proper biopsy involving tumor periphery would help definitive identification of the tumor. Invasive growth at a lesion's periphery, extension into non-neoplastic glands, perineural and lymphovascular invasions all are pathognomonic features of PAC. Immunohistochemistry is mandatory in absence of those diagnostic features. Long-term follow-up surveillance is necessary because recurrences have been reported to occur more than 10 years after initial treatment ${ }^{[6]}$. Among all salivary gland tumors, pleomorphic adenoma (PA) is the most commonly encountered tumor, it accounts for about $60 \%$ of all salivary gland neoplasms. Parotid gland (superficial lobe) is the most common extra-oral site while palate is the most common intra-oral sit of PA. Pleomorphic adenoma presented clinically as rubbery painless mass with 
slow growth rate ${ }^{[7]}$. Histologically, it is greatly variable in appearance hence the name pleomorphic adenoma. Characteristically it has a biphasic appearance, where a mixture of polygonal epithelial cells and spindle-shaped myoepithelial cells in a variable background stroma. Stroma could be cartilaginous, mucoid, myxoid or hyaline. Epithelial cells may be arranged in duct-like structures, sheets, clumps or interlacing cords. The tumor is not capsulated; however it is surrounded by a fibrous pseudocapsule of variable thickness ${ }^{[8]}$. Surgical resection with adequate safety margin is the main treatment modality for salivary gland tumors. Underlying periosteal layer should be included with or without bone resection according to nature of the lesion. Radiotherapy is indicated as an adjuvant therapy if complete resection couldn't be achieved, to improve local recurrence. Generally, irradiation is reserved for inoperable cases.

Co-occurrence of PAC and PA at the same time is very uncommon presentation with accidental detection during radiographic examination. Magnetic resonance imaging (MRI) superiority in detection of concealed soft tissue tumors is the key of diagnosis in such conditions. Perhaps adequate radiographic assessment for both minor and major salivary tissues is mandatory to avoid miss management of such cases. This work has been reported in line with SCARE $2018^{[9]}$.

\section{CASE REPORT}

A 52-year-old male patient visited the maxillofacial surgery outpatient clinic attached to AIN Shams University, complaining of palatal painless swelling over a period of two months. Past medical and surgical history was non-contributory. Social history was significant for 30 years of smoking. No significant family history was reported. Intra -oral clinical examination revealed $3 \times 2 \times 2.5 \mathrm{~cm}$ rubbery well defined mass on the left side of the palate. Covering mucosa had normal looking with small surface ulceration $1 \times 1 \mathrm{~cm}$ (Figure 1). Intra-oral examination for other abnormalities was unremarkable. Clinical examination of related lymph nodes and other salivary glands fail to discover any positive finding. At this point minor salivary gland neoplasm was the preliminary diagnosis and our patient was scheduled for a diagnostic incisional biopsy. Meanwhile magnetic resonance imaging (MRI) examination of the head and neck was requested, the imaging showed $3 \times 3 \times 2 \mathrm{~cm}$ heterogeneous soft tissue mass occupying the left maxilla crossing the midline with cortical impingement of underlying bone (Figure 2). Another well-defined homogenous circular mass $2 \times 2 \mathrm{~cm}$ in superficial lobe of ipsilateral parotid gland was accidentally discovered, however no positive lymph nodes detected (Figure 3).

Incisional biopsy under local anesthesia was done for diagnosis of palatal swelling. Pathology report diagnose the palatal tumor as "polymorphous adenocarcinoma (PAC). Ultra sound (US) guided fine-needle aspiration cytology (FNAC) of parotid mass was done showing a histopathological picture suggestive of pleomorphic adenoma (Figure 4). Work up was done to exclude other primary or synchronous tumors including chest computed tomography (CT) and abdominal ultrasound.

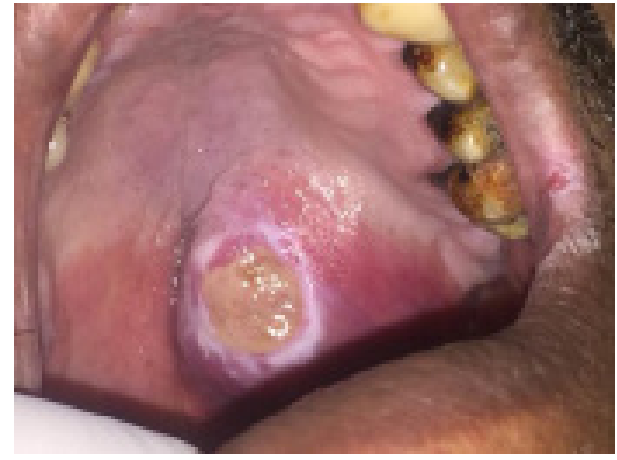

Figuer 1. Intra oral clinical photograph showing $3 \times 3 \mathrm{~cm}$ tumor mass on the left side at the junction of hard and soft palate with central superficial ulceration.

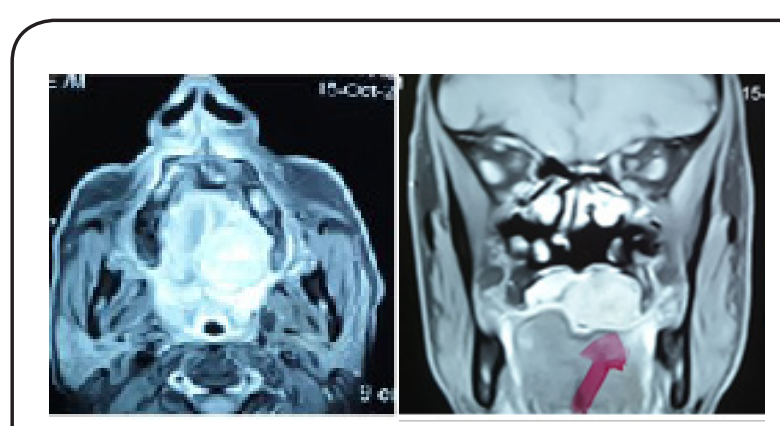

Figure 2. Axial and coronal MRI of palatal mass showing well defined homogenous mass with marked palatal bone resorption.

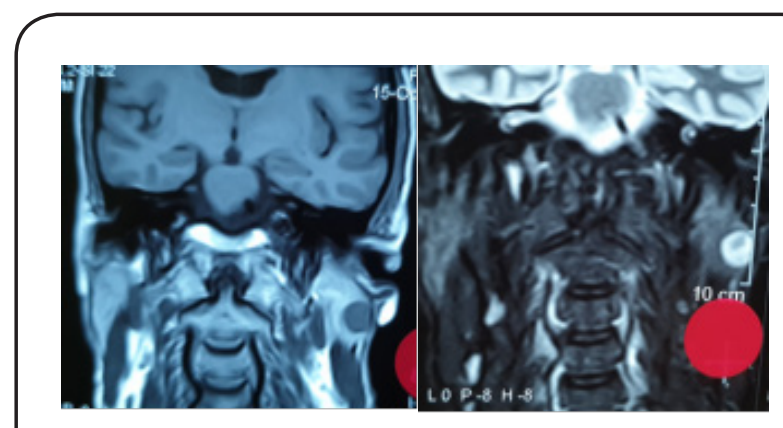

Figure 3. Coronal MRI of parotid gland showing well circumscribed $2 \times 2 \mathrm{~cm}$ mass in superficial lobe accidentally discovered.

The decision was made to surgically resect the palatal tumor with one centimeter safety margin including the underlying palatine bone and insertion of immediate obturator (Figure 5). For synchronously discovered parotid tumor superficial parotidectomy with facial nerve preservation was done 

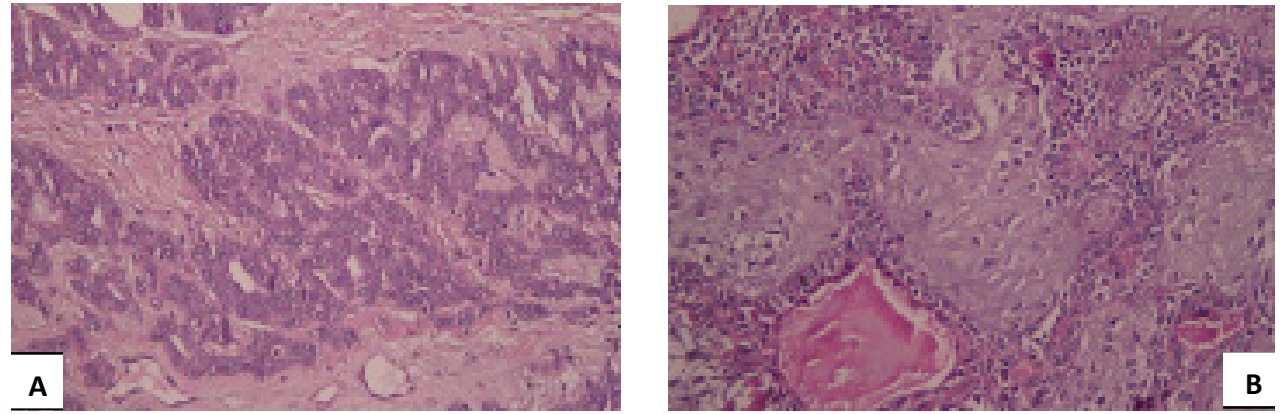

Figure 4. (A) Polymorphous low-grade adenocarcinoma with showing cellular structure eosinophilic cytoplasm and finely dispersed nuclear chromatin. (Hematoxylin and eosinoPhil; original magnification 40X.) (B) Benign pleomorphic adenoma (PA): showing tubular and cribiform growth patterns. No mitoses or necrosis is seen. (Hematoxylin and eosino; original magnification 40X.)
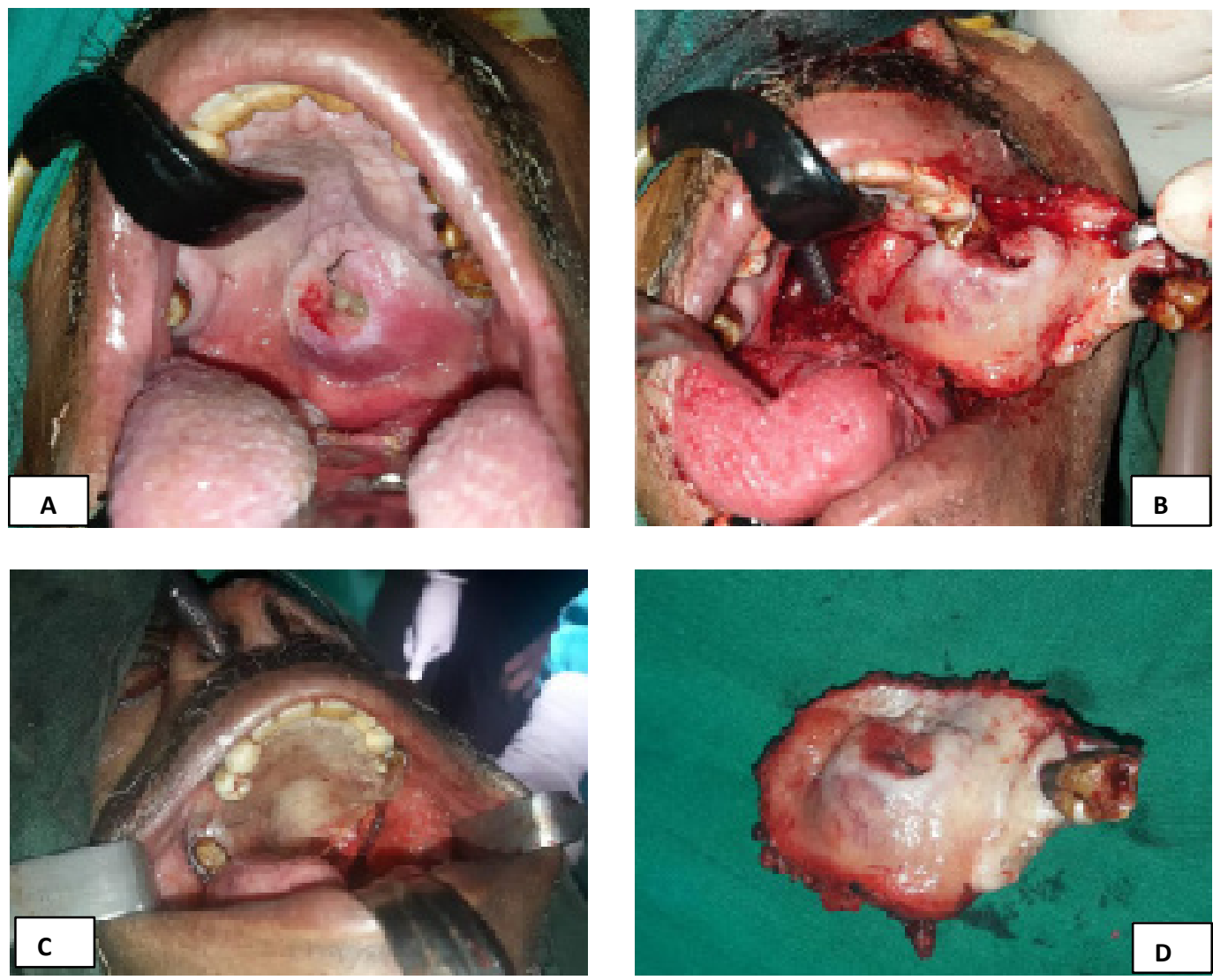

Figure 5. (A) Intra oral clinical photograph showing 3x3 tumor mass diagnosed as (PLGA). (B) Resection of the tumor with one $\mathrm{cm}$ safety margin. (C) Intra-operative clinical photograph showing placement of immediate opturator after resection of palatal lesion. (D) Tumor mass resected with safety margin.

through modified Blair incision followed by primary closure (Figure6). Operative time was five hours with blood loss about 250-350mm, no intra or post-operative complications were recorded. Patient was discharged two days after operation.
Treatment plane was explained to the patient to ensure his acceptance before obtaining informed consent. Resected palatal mass showed perineural invasion (as testified by pathology report) accordingly the patient was scheduled for postoperative 
radiotherapy to control perineural invasion of greater palatine nerve. External beam radiation 60Gy five days per week for six weeks was the recommended protocol by radiotherapist. Patient was free from recurrence after follow up for one year with intact facial nerve function. Two weeks after first radiation dose the patient complains of xerostomia, sore throat, burning mouth, hoarseness, dysphagia, pain, and marked limitation in mouth opening. However, these side effects diminished after two months except for xerostomia.

\section{DISCUSSION}

high-grade transformation has been described ${ }^{[14]}$.

Radiological examination is mandatory in salivary tumors workup. Although ultra sound US is useful in imaging of superficial masses it neither detects malignant features nor invasion of surrounding tissues ${ }^{[13]}$. The next step in diagnosis is biopsy; FNAC is most commonly adopted technique for diagnosis of parotid tumors. It is a quick and safe sampling technique that can be performed readily in the outpatient setting with high specificity and diagnostic accuracy (89\% and $85 \%$ ). However it is technique sensitive. Sensitivity for detecting malignancy has been reported between $70 \%$ and $80 \%$. The diagnostic accuracy can be increased significantly
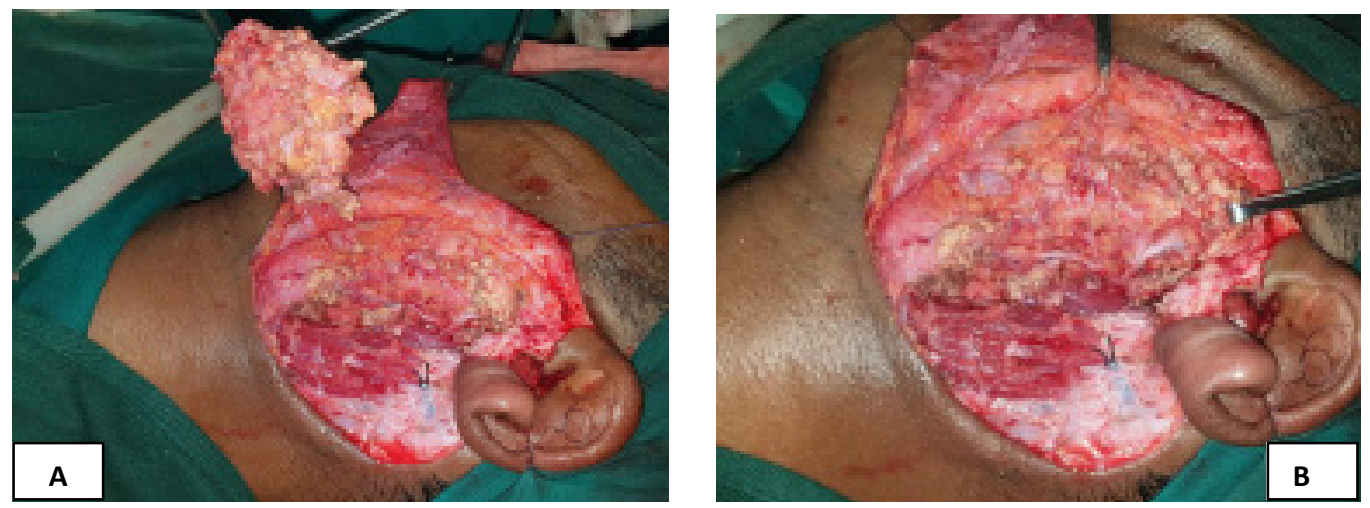

Figure 6. (A) Intra-operative clinical photograph showing exposure and dissection of (PA) polymorphic adenoma using a modified Blair's incision (B) With preservation of facial nerve.

Although salivary gland neoplasms remain idiopathic, there are many risk factors reported in literature including radiation, genetic predilection, excessive smoking, certain oncogenic viral infections and exposure to certain chemicals. Excessive exposure to diagnostic or therapeutic radiation has considerable increased risk of salivary gland neoplasia. There is also significant risk for carcinoma of other organs secondary to primary benign or malignant salivary tumors, especially breast, thyroid, bronchus and ovary ${ }^{[10-12]}$. Polymorphous adenocarcinoma (PAC) were the most reported minor salivary tumor in cases of multiple salivary neoplasms. The mean age for patients with multiple malignant salivary gland neoplasms at the time of their first primary tumor was 53 years ${ }^{[13]}$. Both findings are consistent with our case report.

In cases of multiple salivary tumors pathology of each lesion should be addressed separately. If histopathology is similar the possibility of there being one primary tumor with distant metastasis should be considered ${ }^{[3]}$. In case of different pathology each tumor should be treated independently according to location, size, anatomical extension as well as histological phenotype ${ }^{[4]}$. Recently PAC has been differentiated from a new pathologic variant of salivary tumor named mammary analog secretory carcinoma (MASC). The present case showed clear microscopic features of PAC, in addition there is no conclusive evidence that (MASC) should be treated differently than any other low-grade malignant salivary gland tumors, though when used in conjunction with a cytology and Image guidance. In the present case US was used to guide fine needle aspiration cytology (FNAC) to overcome sampling error ${ }^{[15,16]}$. $\mathrm{CT}$ with contrast is the most commonly used investigation in diagnosis of salivary tumors. In fact MRI is superior in early detection of soft tissue changes and characterization of malignant nodal involvement, detection of underlying bone marrow infiltration as well as anticipation of perineural spread ${ }^{[17]}$. Lymph node involvement in MRI is specified by two criteria: size larger than one centimeter with loss of central fat (central necrosis). The use of MRI rather CT in the present case allows clear detection of coincident parotid lesion that could be missed by CT. None of the examined lymph-nodes showed such criteria accordingly we adopt wait and see policy with frequent clinical and radiographic follow-ups ${ }^{[18,19]}$.

In the present case both lesions were surgically managed in the same operation. Minor salivary tumor PAC was resected with one $\mathrm{cm}$ safety margin including underlying palatal bone to ensure tumor free margins as recommended by literature ${ }^{[13]}$. Insertion of immediate opturator after resection of PAC was decided since it allows frequent examination of the surgical bed for detection of tumor recurrence. Superficial parotidectomy with facial nerve dissection and preservation was the treatment of choice for benign $\mathrm{PA}^{[6]}$. It has been reported in literature that simple enucleation of PA is associated with high recurrence rates ( $8 \%$ and $45 \%$ ), which is reduced to less 
than $5 \%$ with superficial parotidectomy and $0.4 \%$ with total parotidectomy. this was attributed to presence of microscopic extensions protruding beyond pseudocapsule or by capsular penetration ${ }^{[20,21]}$. Unfortunately histopathological evidence of perineural invasion of PAC (through greater palatine nerve) mandate radiation therapy which adds to patient disability. Kämmerer et al, in 2009 report a case of polymorphous lowgrade adenocarcinoma misdiagnosed by incisional biopsy as PA, excisional biopsy later on revealed areas of small malignant fractions of the specimen besides a major part of benign tissue formations. Our case could be a logic explanation of such contribution. Where palatal tumor started as PA and transformed gradually into PAC. By this explanation both tumor would have same pathology at initial presentation ${ }^{[22]}$. We aim to report a rare incidence of multiple primary synchronous salivary tumors of different pathology. Where, the use MRI in diagnosis of salivary tumors is mandatory to detect even early small coincident salivary tissue involvements.

\section{CONCLUSIONS}

The use of MRI examination is mandatory for exclusion of multiple salivary tissue involvement especially if patient has risk factors for malignancy (e.g.: smoking, old age).

\section{ETHICAL APPROVAL AND CONSENT TO PARTICIPATE}

Ethical approval has been exempted by faculty ethical committee based on absence of any risk or violation of applied guidelines in such cases.

\section{CONFLICTS OF INTEREST}

All authors declared that there are no conflicts of interest.

\section{REFERENCES}

1. Licitra, L. , Grandi, C. , Prott, F. J. , Schornagel, J. H. , Bruzzi, P. , \& Molinari, R. . (2003). Major and minor salivary glands tumours. Critical Reviews in Oncology/hematology, 45(2), 215-225.

2. Clayton, J. R. , Pogrel, M. A. , \& Regezi, J. A. . (1995). Simultaneous multifocal polymorphous low-grade adenocarcinoma. report of two cases. Oral Surgery Oral Medicine Oral Pathology Oral Radiology \& Endodontology, 80(1), 71-77.

3. Luz María Ruíz-Godoy R, Mosqueda-Taylor, A. , Lourdes Suárez-Roa, Poitevin, A. , Esther Bandala-Sánchez, \& Abelardo Meneses-García. (2003). Hybrid tumours of the salivary glands. a report of two cases involving the palate and a review of the literature. European Archives of OtoRhino-Laryngology, 260(6), 312-315.

4. Seifert, G. , \& Donath, K. . (1996). Multiple tumours of the salivary glands-terminology and nomenclature. European Journal of Cancer Part B Oral Oncology, 32(1), 0-7.

5. Turk, A. T. , \& Wenig, B. M. . (2014). Pitfalls in the biopsy diagnosis of intraoral minor salivary gland neoplasms. Advances In Anatomic Pathology, 21(1), 1-11.

6. Vincent, S. D. ,Hammond, H.L., \& Finkelstein, M.W..(1994). Clinical and therapeutic features of polymorphous lowgrade adenocarcinoma. Oral Surgery Oral Medicine \& Oral Pathology, 77(1), 41.

7. Johnson, J. T., Ferlito, A., Fagan, J. J., Bradley, P. J. , \& Rinaldo, A. . (2007). Role of limited parotidectomy in management of pleomorphic adenoma. The Journal of Laryngology \& Otology, 121(12), 1126-1128.

8. Ito, F. A. , Jorge, J. , Vargas, P. A. , \& Lopes, M. A. . (2009). Histopathological findings of pleomorphic adenomas of the salivary glands. Medicina Oral Patología Oral Y Cirugía Bucal, 14(2), E57.

9. Agha R.A., Borrelli M.R., Farwana R., Koshy K., Fowler A., Orgill D.P. .(2018).The SCARE 2018 statement: Updating consensus Surgical CAse REport (SCARE) guidelines. International Journal of Surgery,60,132-136.

10. Auclair, P. L. (1991). Salivary gland neoplasms: general considerations. Surgical pathology of the salivary glands, 135-164.

11. Schneider, A. B., Favus, M. J., Stachura, M. E., Arnold, M. J., \& Frohman, L. A. (1977). Salivary gland neoplasms as a late consequence of head and neck irradiation. Ann Intern Med, 87(2), 160-4.

12. Horn-Ross, P. L., Ljung, B. M., \& Morrow, M. (1997). Environmental factors and the risk of salivary gland cancer. Epidemiology, 414-419.

13. Clayton, J. R., Pogrel, M. A., \& Regezi, J. A. (1995). Simultaneous multifocal polymorphous low-grade adenocarcinoma: Report of two cases. Oral Surgery, Oral Medicine, Oral Pathology, Oral Radiology, and Endodontology, 80(1), 71-77.

14. Boliere, C., Murphy, J., Qaisi, M., Manosca, F., \& Fung, H. (2019). Mammary Analogue Secretory Carcinoma of the Palate: Case Report and Review of the Literature. Case reports in dentistry, 2019.

15. Orell, S. R. (1995). Diagnostic difficulties in the interpretation of fine needle aspirates of salivary gland lesions: the problem revisited. Cytopathology, 6(5), 285300.

16. Haldar, S., Sinnott, J. D., Tekeli, K. M., Turner, S. S., \& Howlett, D. C. (2016). Biopsy of parotid masses: review of current techniques. World journal of radiology, 8(5), 501.

17. Lee, Y. Y. P., Wong, K. T., King, A. D., \& Ahuja, A. T. (2008). Imaging of salivary gland tumours. European journal of radiology, 66(3), 419-436.

18. van den Brekel, M. W. (2000). Lymph node metastases: CT and MRI. European journal of radiology, 33(3), 230-238.

19. Moonis, G. , Patel, P. , Koshkareva, Y. , Newman, J. , \& Loevner, L.A.. (2007). Imaging characteristics of recurrent pleomorphic adenoma of the parotid gland. Ajnr Am J Neuroradiol, 28(8), 1532-1536.

20. Park, S.Y., Han, K. T., Kim, M. C., \& Lim, J.S. (2016). Recurrent 
Published online: 29 September 2019

pleomorphic adenoma of the parotid gland. Archives of Craniofacial Surgery, 17(2), 90-92.

21. Zbären P. , Poorten, V. V. , Witt, R. L. , Woolgar, J. A. , Shaha, A. R. , \& Triantafyllou, A. , et al. (2013). Pleomorphic adenoma of the parotid: formal parotidectomy or limited surgery?. American journal of surgery, 205(1),108-119.
22. Kämmerer, P. W., Kreft, A., Toyoshima, T., Al-Nawas, B., \& Klein, M. O. (2009). Misleading initial histological diagnosis of a polymorphous low-grade adenocarcinoma in situ ex pleomorphic adenoma-a case report. Oral and maxillofacial surgery, 13(2), 99-103. 